\title{
Log-aesthetic curves and Riccati equations from the viewpoint of similarity geometry
}

\author{
Masayuki Sato ${ }^{1}$ and Yasuhiro Shimizu ${ }^{2}$ \\ ${ }^{1}$ Serio Corporation, 1-4-8 Shinkawa, Chuo-ku, Tokyo 104-0033, Japan \\ ${ }^{2}$ UEL Corporation, 1-1-1 Toyosu, Koto-ku, Tokyo 135-8560, Japan \\ E-mail masayuki_sato@serio-toyo.co.jp
}

Received November 12, 2014, Accepted December 10, 2014

\begin{abstract}
A family of curves with monotone curvature called the log-aesthetic curves (LAC) has been investigated in the field of industrial shape design. LAC has a radius of curvature in proportional to the power of a linear function of an arc-length parameter. In the present article we show that LAC can be naturally formulated in the similarity geometry and the Riccati equations satisfied by the similarity curvatures of LAC can be derived. Moreover, we clarify that certain generalizations of LAC (GLAC) can also be described in a uniform way.
\end{abstract}

Keywords log-aesthetic curve, similarity geometry, similarity curvature, Riccati equations, generalized log-aesthetic curve

Research Activity Group Applied Integrable Systems

\section{Introduction}

In industrial shape design, various curves with monotone curvature are applied. Among them a family of curves called the log-aesthetic curves has been well investigated with respect to the relation between designers' visual languages and mathematical properties. In the present article we clarify that the log-aesthetic curves are naturally formulated by the notion of the similarity geometry and are described by the Riccati equations in a uniform way. As a result of these arguments, certain generalizations of the log-aesthetic curves are discussed by using the evolutes and the involutes.

The earlier research of this family of curves was made by Harada et al. [1-3]. Associating each curve with the graph called "logarithmic distribution diagram of curvature" (LDDC), they found that attractive curves drawn by car designers have approximately-linear LDDC and the slope of LDDC can be related to designers' visual languages.

We define LDDC using the mathematical reformulation by Nakano et al. [4] and Miura [5,6]. Let $p(s)=$ $(x(s), y(s))$ be a smooth plane curve with an arc-length $s$ and $\rho(s)$ be its radius of curvature. Then LDDC of $p(s)$ is a curve on the $X Y$ plane defined by

$$
(X(s), Y(s))=\left(\log \rho, \log \left(\left|\frac{d s}{d(\log \rho)}\right|\right)\right) .
$$

Miura $[5,6]$ has mathematically formulated the above family of curves which has strictly linear LDDC with the slope $\alpha$ and derived a general formula for the radius of curvature as follows (here we normalize $\rho=1$ at $s=0$ ):

$$
\rho(s)= \begin{cases}e^{\lambda s} & \alpha=0 \\ (\lambda \alpha s+1)^{1 / \alpha} & \alpha \neq 0\end{cases}
$$

Recently the family of curves with the radius of curva-

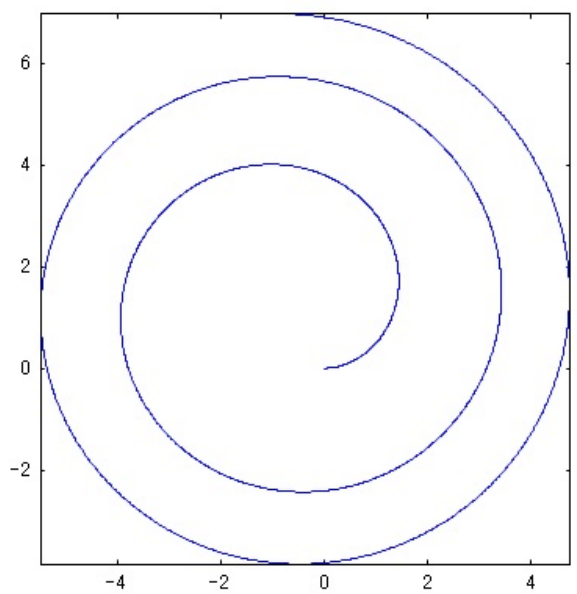

Fig. 1. LAC with $\alpha=3$.

ture defined by (2) is called log-aesthetic curve (LAC). LAC is a family of smooth plane curves with monotone curvature containing the clothoids $(\alpha=-1)$ and the logarithmic spirals $(\alpha=1)$. Fig. 1 gives another example of LAC.

This article is organized as follows. In Section 2, we formulate LAC in terms of the similarity geometry and derive the Riccati equations for the similarity curvatures. In Section 3, we examine the generalizations of LAC by using the Riccati equations satisfied by the similarity curvatures of the evolutes and the involutes of LAC. In Section 4 , future problems are described.

\section{Similarity geometry, log-aesthetic curves and Riccati equations}

At first we briefly review the theory of plane curves in the Euclidean geometry. Let $p(s)$ be a smooth plane curve parametrized by an arc-length $s$ from a base location on the curve. $T(s)=d p / d s$ is a unit tangent 
vector and $N(s)=R(\pi / 2) T(s)$ is a unit normal vector obtained from $T(s)$ where $R(\pi / 2)$ denotes $\pi / 2$ radian rotation in counter-clockwise. The pair of unit vectors $[T(s), N(s)]$ arranged in 2 by 2 matrix manner is called a frame. Differentiating the frame we obtain the Frenet formula

$$
\left[\frac{d T}{d s}(s), \frac{d N}{d s}(s)\right]=[T(s), N(s)]\left[\begin{array}{cc}
0 & -\kappa(s) \\
\kappa(s) & 0
\end{array}\right]
$$

where $\kappa(s)=1 / \rho(s)$ is the curvature and $\rho(s)$ is the radius of curvature.

In the similarity geometry we identify figures overlapped by the similarity transformations in addition to the Euclidean congruent transformations. Since an arclength is not invariant by similarity transformations, we parametrize a curve as $p(\theta)$ by the relative tangent angle $\theta$ from a base location on the curve, which is an invariant parameter in the similarity geometry. Let $T^{\operatorname{sim}}(\theta)=d p / d \theta$ be the similarity tangent vector and $N^{\operatorname{sim}}(\theta)=R(\pi / 2) T^{\operatorname{sim}}(\theta)$ be the similarity normal vector. Here we remark that $T^{\operatorname{sim}}(\theta)$ and $N^{\operatorname{sim}}(\theta)$ have the norm equal to the Euclidean radius of curvature $\rho(s)$ as follows:

$$
\begin{aligned}
T^{s i m}(\theta) & =\frac{d p}{d \theta}=\frac{d s}{d \theta} \frac{d p}{d s} \\
& =\frac{1}{\kappa(s)} T(s)=\rho(s) T(s)
\end{aligned}
$$

and

$$
\begin{aligned}
N^{s i m}(\theta) & =R\left(\frac{\pi}{2}\right) T^{s i m}(\theta) \\
& =\rho(s) R\left(\frac{\pi}{2}\right) T(s)=\rho(s) N(s) .
\end{aligned}
$$

Differentiating the similarity frame $\left[T^{\operatorname{sim}}(\theta), N^{\operatorname{sim}}(\theta)\right]$, we get the similarity Frenet formula

$$
\begin{aligned}
& {\left[\frac{d T^{\operatorname{sim}}}{d \theta}(\theta), \frac{d N^{s i m}}{d \theta}(\theta)\right]} \\
& \quad=\left[T^{\operatorname{sim}}(\theta), N^{\operatorname{sim}}(\theta)\right]\left[\begin{array}{cc}
-S(\theta) & -1 \\
1 & -S(\theta)
\end{array}\right]
\end{aligned}
$$

where

$$
S(\theta)=\frac{1}{\kappa^{2}} \frac{d \kappa}{d s}=-\frac{d \rho}{d s}=-\frac{1}{\rho} \frac{d \rho}{d \theta}
$$

is called the similarity curvature. The similarity curvature of a curve is an invariant in the similarity geometry; see [7, Chapter 13, pp. 120-123] and [8].

We remark that $Y$ axis value of LDDC (1) is given as

$$
Y=X-\log (|S|)
$$

by using the similarity curvature $S$. From (8) we can rewrite the slope $\alpha$ of LDDC only with the notion of the similarity geometry as follows:

$$
\alpha=\frac{d Y}{d X}=1-\frac{1}{S} \frac{d S}{d X}=1+\frac{1}{S^{2}} \frac{d S}{d \theta} .
$$

Thus we conclude that LAC with the slope $\alpha$ of LDDC satisfies the Riccati equation

$$
\frac{d S}{d \theta}=(\alpha-1) S^{2}
$$

which we can solve by quadrature as

$$
S(\theta)=\frac{-\lambda}{(\alpha-1) \lambda \theta+1}=: L(\alpha, \lambda ; \theta) .
$$

From (7) and (11) we can reconstruct the formula for $\rho(\theta)$ of LAC [9] as follows:

$$
\rho(\theta)= \begin{cases}e^{\lambda \theta} & \alpha=1 \\ ((\alpha-1) \lambda \theta+1)^{1 /(\alpha-1)} & \alpha \neq 1 .\end{cases}
$$

\section{Evolute and involute of log-aesthetic curve}

In this section we describe that certain deformations of LAC called the generalized LAC (GLAC) can be examined by using the Riccati equations satisfied by the similarity curvatures of the evolutes and the involutes of LAC. For a smooth plane curve $p(\theta)$ the locus of the center of curvature $\hat{p}(\theta)$

$$
\hat{p}(\theta)=p(\theta)+\rho(\theta) N(\theta)
$$

is called the evolute of $p(\theta)$. In contrast, $p(\theta)$ is called the involute of $\hat{p}(\theta)$; see [10, Appendix B-1, pp. 175-180] and [11, pp. 99-107]. Here we remark that the notion of the evolute is compatible with similarity geometriy, because we have

$$
\hat{p}(\theta)=p(\theta)+N^{s i m}(\theta)
$$

from (5). Differentiating (14) by $\theta$, we get

$$
\begin{aligned}
\frac{d \hat{p}}{d \theta} & =\frac{d p}{d \theta}+\frac{d N^{s i m}(\theta)}{d \theta} \\
& =T^{s i m}+\left(-T^{s i m}-S(\theta) N^{s i m}\right) \\
& =-S(\theta) N^{s i m}
\end{aligned}
$$

by using the similarity Frenet formula (6) to $p(\theta)$. Hence the tangent angle parameter $\hat{\theta}$ of the evolute is

$$
\hat{\theta}=\theta \pm \frac{\pi}{2}
$$

Then the similarity tangent vector $\hat{T}^{\operatorname{sim}}(\hat{\theta})$ of $\hat{p}(\hat{\theta})$ is

$$
\hat{T}^{\operatorname{sim}}(\hat{\theta})=\frac{d \hat{p}}{d \hat{\theta}}=\frac{d \hat{p}}{d \theta}=-S(\theta) N^{s i m},
$$

and the similarity normal vector $\hat{N}^{\operatorname{sim}}(\hat{\theta})$ is

$$
\begin{aligned}
\hat{N}^{\operatorname{sim}}(\hat{\theta}) & =R\left(\frac{\pi}{2}\right) \hat{T}^{\text {sim }}(\hat{\theta}) \\
& =(-S(\theta))\left(-T^{s i m}\right)=S(\theta) T^{s i m} .
\end{aligned}
$$

Differentiating (17) with respect to $\hat{\theta}$ gives

$$
\begin{aligned}
\frac{d \hat{T}^{s i m}}{d \hat{\theta}}(\hat{\theta}) & =-\left(\frac{d S}{d \theta} N^{s i m}+S \frac{d N^{s i m}}{d \theta}\right) \\
& =-\frac{d S}{d \theta} N^{s i m}-S\left(-T^{s i m}-S N^{s i m}\right) \\
& =\left(\frac{d S}{d \theta}-S^{2}\right)\left(-N^{s i m}\right)+S T^{s i m} \\
& =\left(\frac{\frac{d S}{d \theta}-S^{2}}{S}\right) \hat{T}^{s i m}+\hat{N}^{s i m}
\end{aligned}
$$




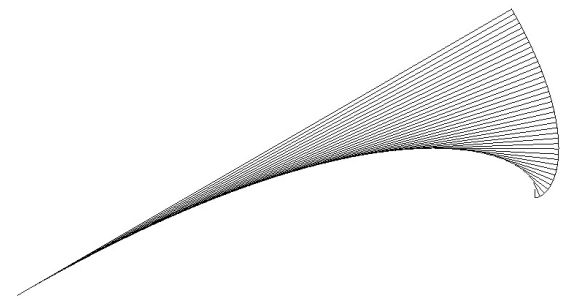

Fig. 2. evolute of LAC.

On the other hand, using the similarity Frenet formula to $\hat{p}(\hat{\theta})$, we get

$$
\frac{d \hat{T}^{s i m}}{d \hat{\theta}}(\hat{\theta})=-\hat{S}(\hat{\theta}) \hat{T}^{s i m}(\hat{\theta})+\hat{N}^{s i m}(\hat{\theta}),
$$

where $\hat{S}(\hat{\theta})$ is the similarity curvature of $\hat{p}(\hat{\theta})$. Comparing (19) with (20) we have

$$
-\hat{S}(\hat{\theta})=\frac{\frac{d S}{d \theta}-S^{2}}{S} .
$$

Eq. (21) can be seen as the Riccati equation satisfied by the similarity curvature $S(\theta)$ of the involute $p(\theta)$ as follows:

$$
\frac{d S}{d \theta}(\theta)=(S(\theta))^{2}-\hat{S}(\hat{\theta}) S(\theta) .
$$

Let $p(\theta)$ be a LAC and $S(\theta)=L(\alpha, \lambda ; \theta)$ be its similarity curvature. We rewrite (10) as follows:

$$
\frac{d S}{d \theta}=S^{2}-((2-\alpha) S) S .
$$

Here $(2-\alpha) S(\theta)=(2-\alpha) L(\alpha, \lambda ; \theta)$ can be seen as the similarity curvature $\hat{S}(\hat{\theta})$ of the evolute $\hat{p}(\hat{\theta})$. If $\alpha=$ $2,(2-\alpha) S(\theta)=0$ is the similarity curvature of a circle, so that the evolute $\hat{p}(\hat{\theta})$ is a circle. If $\alpha \neq 2$, we can show that the evolute of LAC is also LAC (Fig. 2) and its slope of LDDC is $1 /(2-\alpha)$ as follows :

$$
\begin{aligned}
\hat{S}(\hat{\theta}) & =(2-\alpha) L\left(\alpha, \lambda ; \hat{\theta}-\frac{\pi}{2}\right) \\
& =\frac{(2-\alpha)(-\lambda)}{(\alpha-1) \lambda\left(\hat{\theta}-\frac{\pi}{2}\right)+1} \\
& =\frac{-(2-\alpha) \lambda}{\left(\frac{1}{2-\alpha}-1\right)(2-\alpha) \lambda\left(\hat{\theta}-\frac{\pi}{2}\right)+1} \\
& =L\left(\frac{1}{2-\alpha},(2-\alpha) \lambda ; \hat{\theta}-\frac{\pi}{2}\right) .
\end{aligned}
$$

Eq. (23) and (24) give an alternative proof of the result by Yoshida et al. [12].

One can trace back the previous arguments. If $\alpha \neq$ 1,2 , we take the Riccati equation

$$
\frac{d S}{d \theta}(\theta)=(S(\theta))^{2}-L\left(\frac{1}{2-\alpha},(2-\alpha) \lambda ; \theta\right) S(\theta),
$$

which is satisfied by the involute of LAC with the similarity curvature

$$
\hat{S}(\hat{\theta})=L\left(\frac{1}{2-\alpha},(2-\alpha) \lambda ; \hat{\theta}-\frac{\pi}{2}\right) .
$$

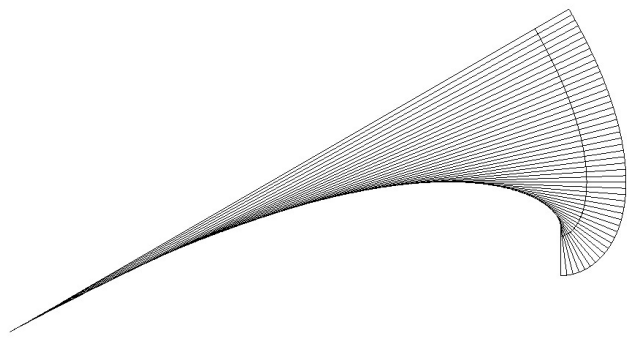

Fig. 3. Involute of LAC.

Noting that $S(\theta)=L(\alpha, \lambda ; \theta)$ is a special solution of (25), we obtain a general solution of (25)

$$
S(\theta)=\frac{L(\alpha, \lambda ; \theta)}{1+\frac{1}{C \lambda((\alpha-1) \lambda \theta+1)^{1 /(\alpha-1)}}},
$$

where $C$ is an integral constant [13, chapter 11, pp.147160]. Here we remark that the preceding solution coincides with the similarity curvature of $\rho$-shift GLAC (generalized log-aesthetic curve). Miura et al. [14, 15] proposed the generalizations of LAC i.e. GLAC. One of GLAC is called $\rho$-shift GLAC, which has the radius of curvature $\tilde{\rho}(\theta)$ shifted by a constant $\nu$ from $\rho(\theta)$ of LAC:

$$
\tilde{\rho}(\theta)=((\alpha-1) \lambda \theta+1)^{1 /(\alpha-1)}+\nu
$$

Calculating the similarity curvature $\tilde{S}(\theta)$ of $\rho$-shift GLAC, we get

$$
\begin{aligned}
\tilde{S}(\theta) & =-\frac{\frac{d \tilde{\rho}}{d \theta}}{\tilde{\rho}} \\
& =-\frac{\lambda((\alpha-1) \lambda \theta+1)^{1 /(\alpha-1)-1}}{((\alpha-1) \lambda \theta+1)^{1 /(\alpha-1)}+\nu} \\
& =\frac{(-\lambda)((\alpha-1) \lambda \theta+1)^{-1}}{1+\nu((\alpha-1) \lambda \theta+1)^{-1 /(\alpha-1)}} \\
& =\frac{L(\alpha, \lambda ; \theta)}{1+\frac{\nu}{((\alpha-1) \lambda \theta+1)^{1 /(\alpha-1)}}} .
\end{aligned}
$$

Substituting $\nu=1 /(C \lambda)$ in $(29)$, we conclude that $\rho$ shift GLAC can be seen as an involute of LAC (Fig. 3).

\section{Future problems}

In the present article, only the Riccati equations of Bernoulli type appeared. However, the similarity curvatures of some curves, which appear in Harada et al. [1-3], satisfy the Riccati equations of non-Bernoulli type. In the strict sense their LDDC are not linear, but are approximately or asymptotically linear. We will investigate the family of curves in a neighborhood of LAC from the viewpoint of the similarity geometry in the forthcoming publications.

\section{Acknowledgments}

The authors would like to thank Professor Jun-ichi Inoguchi for useful suggestions about the similarity geometry. We also express thanks to Professor Kenji Kajiwara for his kind advice. 


\section{References}

[1] T. Harada, N. Mori and K. Sugiyama, Study of quantitative analysis of curve's character (in Japanese), Bulletin of JSSD, 40 (1994), 9-16.

[2] T. Harada, Study of quantitative analysis of the characteristics of a curve, Forma, 12 (1997), 55-63.

[3] T. Harada, F. Yoshimoto and M. Moriyama, An aesthetic curve in the field of industrial design, in: VL '99 Proceedings of the IEEE Symposium on Visual Languages, pp. 38-47, IEEE Computer Society Press, New York, 1999.

[4] Y. Nakano, I. Kanaya, K. Sato and S. Inokuchi, Sensuous evaluation of aesthetic surface based on 3D curvature profile (in Japanese), in: Proc. of the 2003 IEICE general conference, D-12-17, p. 178, 2003.

[5] K. T. Miura, General formulas of aesthetic curves (in Japanese), in: Proc. of VC/GCAD joint symposium 2005, pp. $227-232,2005$.

[6] K. T. Miura, A general equation of aesthetic curves and its self-affinity, Comput.-Aided Design Appl., 3 (2006), 457-464.

[7] J. Inoguchi, Curves and Solitons (in Japanese), Asakura Publishing, Tokyo, 2010.

[8] K.-S. Chou and C. Qu, Motions of curves in similarity geometries and Burgers-mKdV hierarchies, Chaos Soliton. Fract., 19 (2004), 47-53.

[9] N. Yoshida and T. Saito, Interactive aesthetic curve segments, The Visual Computer (Proc. of Pacific Graphics), 22 (2006), 896-905.

[10] M. Umehara and K. Yamada, Curves and Surfaces (in Japanese), SHOKABO, Tokyo, 2002.

[11] A. Gray, E. Abbena and S. Salamon, Modern Differential Geometry of Curves and Surfaces with Mathematica, 3rd ed., Chapman and Hall/CRC, London, 2006.

[12] N. Yoshida and T. Saito, On the evolutes of log-aesthetic planar curves (in Japanese), Research Report A of College of Industrial Technology, Nihon University, 44 (2011), 1-5.

[13] J. Inoguchi, Secrets of Riccati Equations (in Japanese), Nippon Hyoron Sha, Tokyo, 2010.

[14] K. T. Miura and R. U. Gobithaasan, Generalized log-aesthetic curve: GLAC (in Japanese), in: Proc. of VC/GCAD joint symposium 2009, pp. 10_1-10_6, 2009.

[15] R. U. Gobithaasan and K. T. Miura, Aesthetic spiral for design, Sains Malaysiana, 40 (2011), 1301-1305. 